\title{
Overexpression of long non-coding RNA MFI2 promotes cell proliferation and suppresses apoptosis in human osteosarcoma
}

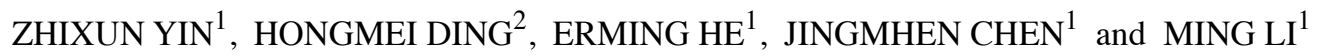 \\ ${ }^{1}$ Department of Orthopaedic Surgery, The First Affiliated Hospital of Guangzhou Medical University, Guangzhou, \\ Guangdong 510120; ${ }^{2}$ Department of Anatomy, Guangzhou Medical University, Guangzhou, Guangdong 510182, P.R. China
}

Received March 14, 2016; Accepted July 18, 2016

DOI: $10.3892 /$ or.2016.5013

\begin{abstract}
The long non-coding RNA MFI2 antisense RNA is overexpressed in human cancer tissues and its increased expression is associated with occurrence and metastasis of cancer. However, the underlying mechanism in evolution and progression of human osteosarcoma is not well known. In the present study, we aimed to evaluate the molecular mechanism of IncRNA MFI2 in promoting osteosarcoma cell proliferation and suppressing apoptosis. We found that the IncRNA MFI2 was significantly overexpressed in human osteosarcoma tissues. Knockdown of IncRNA MFI2 expression suppressed MG63 and SAOS-2 cell proliferation, migration and invasion, and induced cell apoptosis. Furthermore, the expression of forkhead box P4 (FOXP4) was significantly increased and it was positively associated with lncRNA MFI2 expression in tumor tissues. In addition, knockdown of FOXP4 expression by RNA interference strategy inhibited osteosarcoma cell proliferation, migration and invasion, and promoted cell apoptosis. All the results indicated lncRNA MFI2 could promote proliferation and migration of osteosarcoma cells by regulating FOXP4 expression, which suggested critical roles of IncRNA MFI2 and FOXP4 in occurrence and development of human osteosarcoma.
\end{abstract}

\section{Introduction}

Osteosarcoma is one of the most common human primary bone cancers in children and young adults, which mainly presents around the active bone growth and reparation regions, and accounts for nearly $15 \%$ of all bone cancers (1). In the recent 25 years, the incidence of osteosarcoma increased $1.4 \%$ per year (2). Osteosarcoma is not only locally destructive, but it also has a high metastatic potential (3). At present, the standard

Correspondence to: Professor Hongmei Ding, Department of Anatomy, Guangzhou Medical University, 195 Dongfeng Xi Road, Guangzhou, Guangdong 510182, P.R. China

E-mail: dinghongmei110@126.com

Key words: osteosarcoma, long non-coding RNA, MFI2, FOXP4, proliferation, migration and invasion treatment is surgical operation and neoadjuvant chemotherapy. For the last 30 years, the therapy has effectively improved patients survival rate, but poor prognosis still threaten the patients with recurrent or metastatic osteosarcomas $(4,5)$. Therefore, the standard treatment is not only a critical therapy for improving the prognosis of osteosarcoma patients, but it is also important to prevent the pulmonary metastases during the early stage of the tumor. However, this cancer is difficult to diagnose and confirm at the initial stage. The limitations of established osteosarcoma biomarkers require us to identify better molecular target that could be clinically useful for diagnosis and prognosis, in particular, for the high-risk patient groups with high risk of progression, recurrence and metastasis.

As is known, only less than $2 \%$ of the genome serves as the transcription template for protein coding, whereas more than $70 \%$ of the human genes transcripted into non-coding RNAs (6). The non-coding transcripts are named as noncoding RNAs (ncRNAs). The non-coding RNAs are classified as small or long non-coding RNAs (lncRNAs) according to length. IncRNAs are a group of RNAs more than $200 \mathrm{nt}$ in length with limited or no protein-coding capacity $(7,8)$. Most lncRNAs are evolutionarily conserved and strictly regulated, playing critical roles in regulating gene expression (9). IncRNAs are often dysregulated and are involved in the occurrence and development of human cancers $(10,11)$. Substantial evidence revealed that IncRNAs have tumor suppressor or oncogenic roles in tumorigenesis. It is well known that lncRNA HOTAIR acts as an oncogene involved in tumor pathogenesis. The expression of IncRNA HOTAIR is consistently upregulated and it was identified as a strong prognosis biomarker of patient outcomes, including patient survival and metastasis in various human tumors (12-14). IncRNA MALAT1 is an oncogenic lncRNA, which was found overexpressed in several tumor tissues and it controls cell proliferation and cell cycle progression $(15,16)$. IncRNA SNHG12 is an oncogene which is upregulated in human osteosarcoma. Knockdown of SNHG12 suppressed cell growth, invasion and migration (17). Nevertheless, the overall pathophysiological contributions of IncRNAs to osteosarcoma remain unknown. Previous study showed that the expression of 1ncRNA MFI2 was significantly upregulated in human osteosarcoma (18). However, little is known of the exact role of lncRNA MFI2 in evolution and progression of human osteosarcoma. 
In the present study, we first performed quantitative real-time PCR analysis of the expression level of lncRNA MFI2 in human osteosarcoma tissues, and found that the expression of MFI2 was significantly increased in tumor tissues when compared with matched adjacent normal tissues, which is consistent with the result of DNA microarray. However, the role of lncRNA MFI2 in osteosarcoma is poorly known and its functional significance has not yet been established. In the present study, we demonstrated that increased expression of MFI2 was a characteristic molecular change in osteosarcoma. Furthermore, we investigated the effect of knockdown of MFI2 on the proliferation, migration, invasion and apoptosis of osteosarcoma cells in vitro. Our findings suggest that lncRNA MFI2 may represent a novel biomarker in osteosarcoma and may be a potential therapeutic target for diagnosis and therapy.

\section{Materials and methods}

Tissue collection. Between 2014 and 2015, 43 osteosarcoma and matched adjacent normal tissues were obtained from patients who underwent surgery at the First Affiliated Hospital of Guangzhou Medical University, and were diagnosed with osteosarcoma (stages II and III) based on histopathological evaluation according to the International Union for Cancer Control (UICC). No local or systemic treatment was conducted in these patients before the operation. All volunteers provided written consent for the use of their specimens in this study. All tissues were collected and immediately frozen in liquid nitrogen, and stored at $-80^{\circ} \mathrm{C}$ until RNA extraction. The present study was approved by the Research Ethics Committee of the First Affiliated Hospital of Guangzhou Medical University.

Cell lines and culture conditions. Human osteoblasts cell line hFOB (OB3) and human osteosarcoma cell lines, SAOS-2, MG63, and U-2OS were purchased from the American Type Culture Collection (ATCC) (Manassas, VA, USA). The osteoblasts and osteosarcoma cells were maintained according to the vendor's instructions. In brief, SAOS-2 and U-2OS cells were cultured in McCoy's 5A medium (modified) (ATCC). MG-63 cells were cultured in Eagle's minimum essential medium (EMEM) (ATCC). Human osteosarcoma cells were all cultured with $10 \%$ fetal bovine serum (FBS). hFOB (OB3) cells were cultured in a 1:1 mixture of Ham's F12 medium Dulbecco's modified Eagle's medium, with $2.5 \mathrm{mM}$ L-glutamine and 20\% FBS. All medium contained 1\% penicillin-streptomycin $(100 \mathrm{U} / \mathrm{ml}$ penicillin and $100 \mu \mathrm{g} / \mathrm{ml}$ streptomycin). All cells were cultured and maintained in a humidified incubator at $37^{\circ} \mathrm{C}$ and supplemented with $5 \%$ $\mathrm{CO}_{2}$.

RNA extraction and quantitative real-time PCR ( $q R T-P C R)$ analysis. Total RNA was extracted using TRIzol reagent (Invitrogen Inc., Carlsbad, CA, USA). For qRT-PCR, $2 \mu \mathrm{g}$ of total RNA was used for reverse transcription reaction and cDNA synthesis using M-MLV reverse transcriptase (Promega, Madison, WI, USA). Quantitative real-time PCR analyses were performed with SYBR-Green Real-Time Master Mix (Toyobo, Tokyo, Japan). Results were normalized to a constitutive expression gene, GAPDH. The PCR primers for MIF2 were: 5'-CACTGCTGAGACGACATCCCTT-3' (forward) and
5'-GACTTGCTCTTTGGGTCACTGTAT-3' (reverse); for FOXP4, 5'-GCCATCCTGGAAACCCCT-3' (forward) and 5'-CTGATACTCCCGCTCGTC-3' (reverse); for GAPDH, 5'-TGTTCGTCATGGGTGTGAA-3' (forward) and 5'-ATGG CATGGACTGTGGTCAT-3' (reverse). qRT-PCR and data collection were performed on Applied Biosystems 7500 Sequence Detection system (ABI, USA). The relative expression levels of MFI2 and FOXP4 were calculated and normalized using the $2^{-\Delta \Delta \mathrm{Ct}}$ method.

Protein extraction and western blotting. Total proteins were extracted using SDS lysis buffer (Beyotime, Jiangsu, China) on ice for $30 \mathrm{~min}$ and the protein concentration were determined using BCA protein assay kit (Pierce, Rockford, IL, USA). Equal amounts of total proteins were separated by $12 \%$ SDS-polyacrylamide gel electrophoresis (SDS-PAGE), transferred to $0.22 \mu \mathrm{m}$ polyvinylidene fluoride (PVDF) membranes (Millipore, Billerica, MA, USA) and incubated with FOXP4 $(1: 1,000)$ or GAPDH $(1: 1,500)$ antibodies (both from Abcam, Cambridge, MA, USA). Proteins were detected by enhanced chemiluminescence (ECL) as described by the manufacturer (Beyotime), and the intensity of the bands was quantified by densitometry (Quantity One software; Bio-Rad, Hercules, CA, USA).

siRNA transfection. The small interfering RNA (siRNA) targeting MFI2 (5'-AAAUUGUUUAGGUUUGUGGGG-3' and 5'-CCACAAACCUAAACAAUUUCG-3'); FOXP4 (5'-UGUAGAACUCAUGAUUCUGGG-3' and 5'-CAGAAUC AUGAGUUCUACAAG-3'); scrambled sequence negative control (5'-UCUUCCGAACGUGUCACGUTT-3' and 5'-ACR UGACACGUUCGGAGAATT-3') were obtained from GenePharma (Shanghai, China). Osteosarcoma cells were seeded into 24 -well plates $\left(1 \times 10^{5} /\right.$ well) for $24 \mathrm{~h}$. Then, cells were transfected with MFI2-siRNA or FOXP4-siRNA or scrambled sequence (NC) (100 nM) using Lipofectamine 2000 (Invitrogen) in serum-free medium in accordance with the manufacturer's instructions.

Cell viability assay. The cell viability of osteosarcoma cells with siRNA duplexes at 24, 48 and $72 \mathrm{~h}$ was detected using Cell Counting Kit-8 (CCK-8) (Dojindo, Kumamoto, Japan) according to the manufacturer's instructions. In brief, $1 \times 10^{4}$ cells/well were seeded into 96-well tissue culture plates for 24, 48 and $72 \mathrm{~h}$. Then, osteosarcoma cells were treated with CCK- 8 at $37^{\circ} \mathrm{C}$ for $1 \mathrm{~h}$. Osteosarcoma cells was used to measure the absorbency at $450 \mathrm{~nm}$ using a microplate reader Thermo Plate (Rayto Life and Analytical Sciences Co., Ltd., Germany).

Flow cytometry. Cell apoptosis was detected using Annexin V-FITC/PI apoptosis detection kit (Life Technologies, Grand Island, NY, USA) by flow cytometry in accordance with the manufacturer's instructions. Briefly, $5 \times 10^{6}$ cells were harvested and stained using the Annexin V-FITC/PI apoptosis detection kit. Then, samples were analyzed by a FACScan (Becton-Dickinson, San Diego, CA, USA) flow cytometry. Annexin $\mathrm{V}\left({ }^{+}\right) / \mathrm{P}\left({ }^{-}\right)$and Annexin $\left.\mathrm{V}^{+}\right) / \mathrm{P}\left(^{+}\right)$represent the osteosarcoma cells in early apoptosis and late apoptosis/necrosis, respectively. 

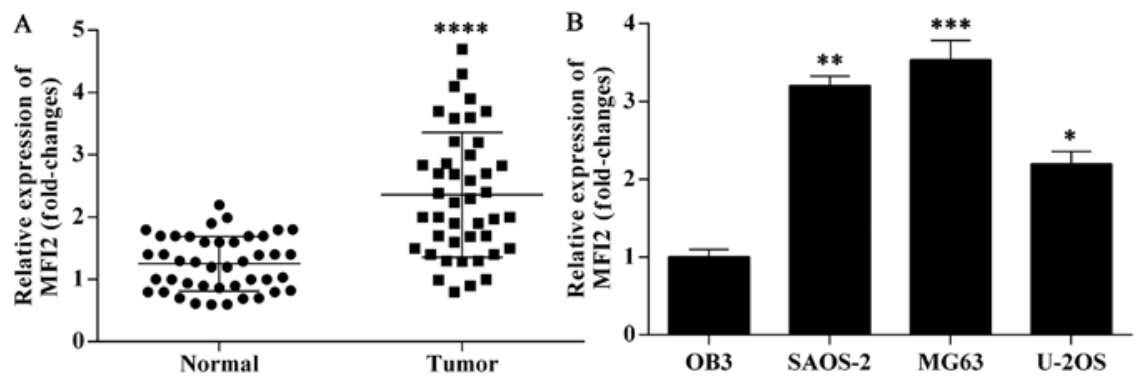

Figure 1. IncRNA MFI2 is upregulated in human osteosarcoma tissues and osteosarcoma cell lines. (A) qRT-PCR revealed that the expression of lncRNA MFI2 was significantly upregulated in osteosarcoma tissues; ${ }^{* * * * *} \mathrm{P}<0.0001$. (B) qRT-PCR analyzed mRNA expression levels of SNHG12 in OB3, SAOS-2, MG-63 and U-2OS cell lins; ${ }^{* * *} \mathrm{P}<0.001,{ }^{* *} \mathrm{P}<0.01,{ }^{*} \mathrm{P}<0.05$.
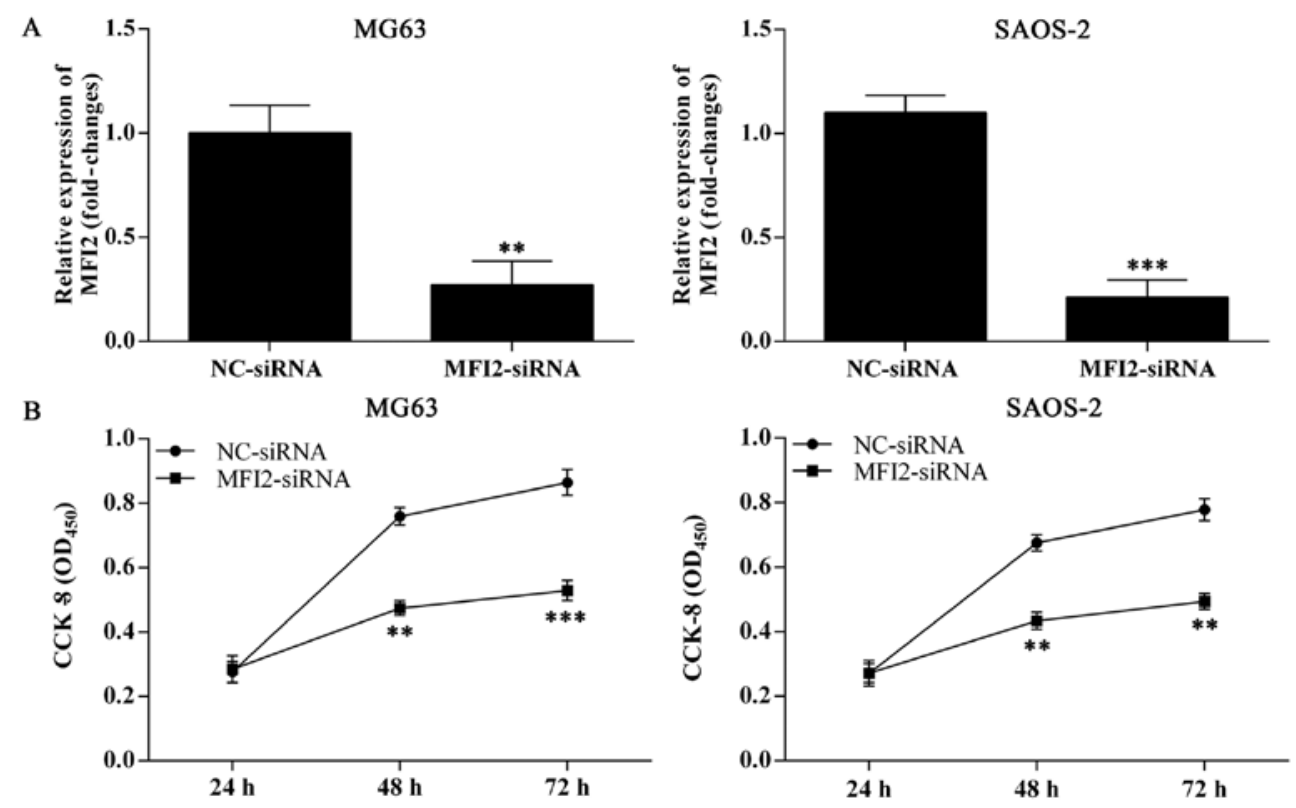

Figure 2. Knockdown of MFI2 suppresses osteosarcoma cell proliferation. (A) The expression of MFI2 in SAOS-2 and MG63 cells transfected with MFI2-siRNA and scramble negative control (NC) siRNA was detected using qRT-PCR; ${ }^{* * *} \mathrm{p}<0.01,{ }^{* * * *} \mathrm{P}<0.001$. (B) CCK-8 assay was used to detect proliferation rates of SAOS-2 and MG63 cells transfected with MFI2-siRNA or NC; ${ }^{* *} \mathrm{P}<0.01,{ }^{* * *} \mathrm{P}<0.001$.

Cell migration and invasion assays. Transwell migration and Matrigel invasion assays were performed using 24-well Transwell inserts with $8-\mu \mathrm{m}$ pore size (Corning Costar Corp., Corning, NY, USA), respectively. For Transwell migration assay, $2 \times 10^{4}$ osteosarcoma cells suspended in $100 \mu 1$ serum-free corresponding culture medium were loaded into upper chamber of Transwell insert with non-coated membrane. For Transwell invasion assay, $3 \times 10^{4}$ osteosarcoma cells were plated in $100 \mu \mathrm{l}$ corresponding culture medium without FBS in the upper Matrigel-coated chamber instead. In both assays, $500 \mu \mathrm{l}$ of culture medium containing $20 \%$ FBS was added to the lower chamber for culture. The cells were cultured in a humidified atmosphere for $24 \mathrm{~h}$ at $37^{\circ} \mathrm{C}$ and $5 \%$ $\mathrm{CO}_{2}$, and the non-invading cells were removed. The migrated or invaded cells were fixed with $100 \%$ methanol for $30 \mathrm{~min}$ and stained using $0.5 \%$ crystal violet (Sigma, St. Louis, MO, USA) for $20 \mathrm{~min}$, and the permeating cells were counted under a phase-contrast microscope (Olympus, Tokyo, Japan).

Statistical analysis. The SPSS 20.0 program (SPSS, Inc., Chicago, IL, USA) was used for statistical analyses. For quantitative data, all experiments were performed at least three times. All data are expressed as the mean \pm SD. One-way analysis of variance (ANOVA) or a paired Student's t-test was used to statistical analysis. To assess interaction between the variables, the MFI2 expression level was cross-correlated with FOXP4 in the univariate Cox model, and interaction was evaluated by the Wald test. P-value of $<0.05$ was considered to indicate a statistically significant result.

\section{Results}

IncRNA MFI2 is upregulated in human osteosarcoma tissues and osteosarcoma cell lines. To investigate the expression level of MFI2 in human osteosarcoma tissues, qRT-PCR was used to examine MFI2 expression in 43 paired osteosarcoma samples and matched adjacent normal tissues. As shown in Fig. 1A, MFI2 was significantly upregulated in human osteosarcoma tissues compared with corresponding adjacent, histological normal tissues $(\mathrm{P}<0.0001)$. In addition, the expression level of MFI2 was also detected in osteosarcoma cell lines. The result showed that MFI2 was significantly upregulated in 
A

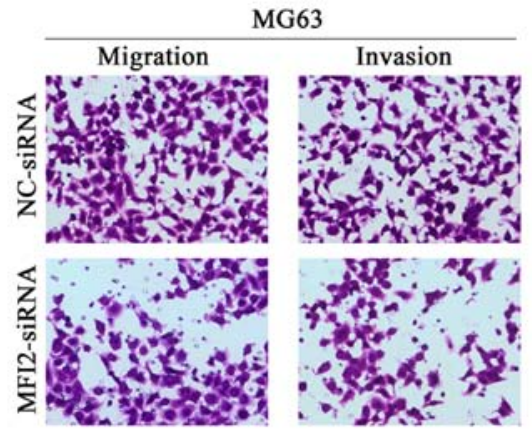

B

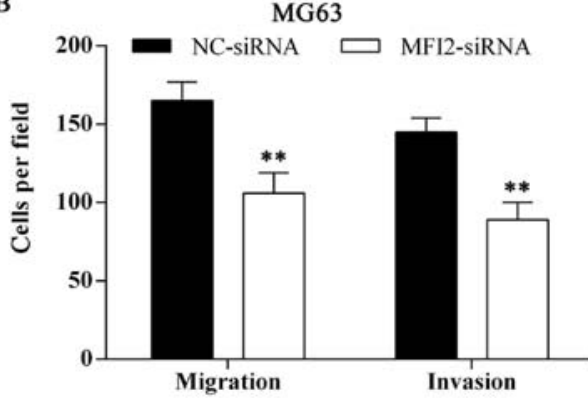

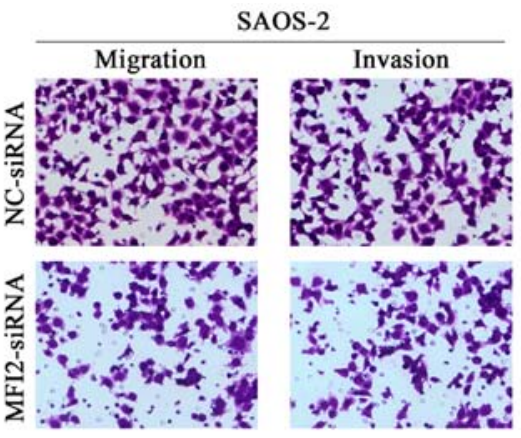

SAOS-2

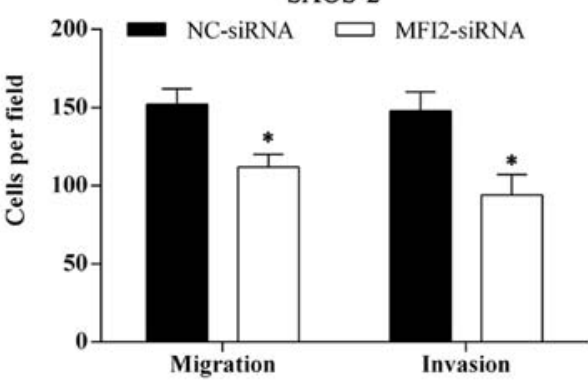

Figure 3. Knockdown of MFI2 inhibits osteosarcoma cell migration and invasion. (A) Transwell assay was performed to detec T cell migration and invasion (magnification, x40). Images of migration and invasion ability of each group are presented. (B) The average migration and invasion cell number/field among different experimental groups. Data are the mean $\pm \mathrm{SD}$ from three independent experiments; ${ }^{*} \mathrm{P}<0.05 ;{ }^{* *} \mathrm{P}<0.01$.

A

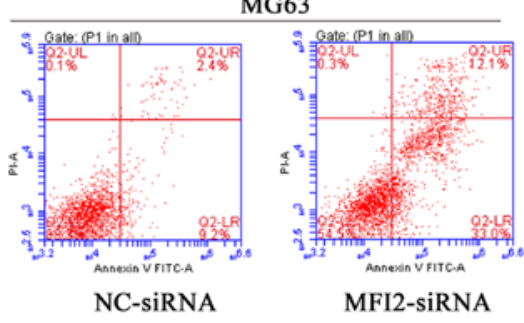

B

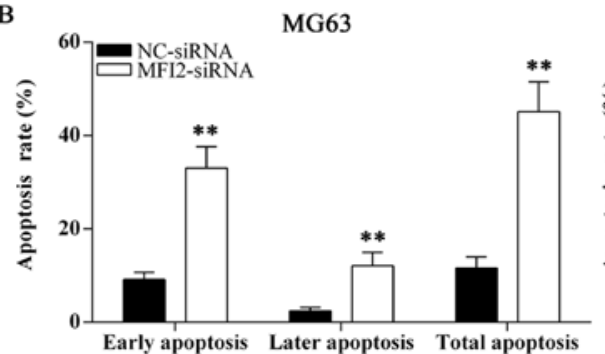

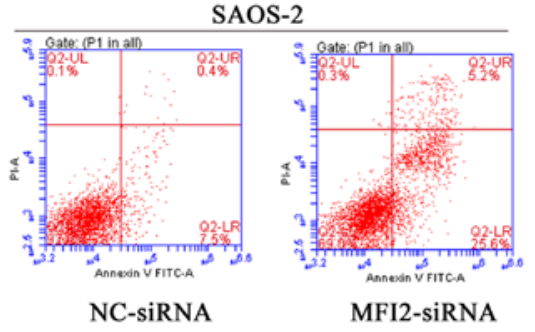

SAOS-2

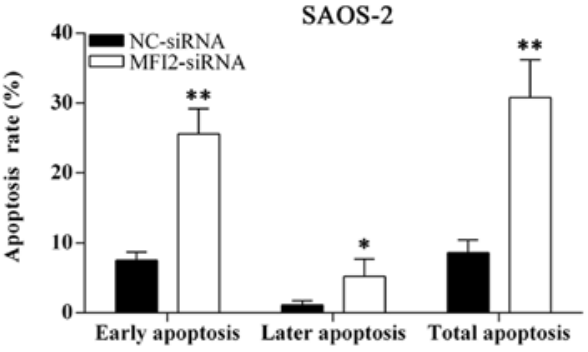

Figure 4. Knockdown of MFI2 promotes osteosarcoma cell apoptosis. Cell death was monitored by Annexin V-FITC/PI staining and flow cytometry. (A) Flow cytometric analysis of SAOS-2 and MG63 cells in different experimental groups. (B) The percentage of cells in early, later and total apoptosis of each group. Data are from three independent experiments; ${ }^{*} \mathrm{P}<0.05 ;{ }^{* *} \mathrm{P}<0.01$. LR (lower right), early apoptosis cells; UR (upper right), late apoptosis cells; LL (lower left), viable cells; UL (upper left), necrosis cells.

osteosarcoma cells (Fig. 1B). Based on the above results, osteosarcoma cell lines SAOS-2 and MG63 were selected for the present study.

Knockdown of MFI2 suppresses osteosarcoma cell proliferation. To evaluate the impact of MFI2 on osteosarcoma cell proliferation, MFI2 was knocked down by MFI2-siRNA transfection. MFI2 was significantly decreased in MG63 and SAOS-2 cells after MFI2-siRNA transfection (Fig. 2A). After transfected with MFI2-siRNA, cell proliferation was performed using CCK- 8 assay. The results showed osteosarcoma cell proliferation was significantly suppressed by knockdown of MFI2 (Fig. 2B).

Knockdown of MFI2 inhibits osteosarcoma cell migration and invasion in vitro. To assess the role of MFI2 on tumor cell migration and invasion, Transwell assay was used to detect the migration and invasion ability of MG63 and SAOS-2 cells after MFI2-siRNA transfection. Knockdown of MFI2 inhibited the migration and invasion of MG63 and SAOS-2 cells (Fig. 3). 

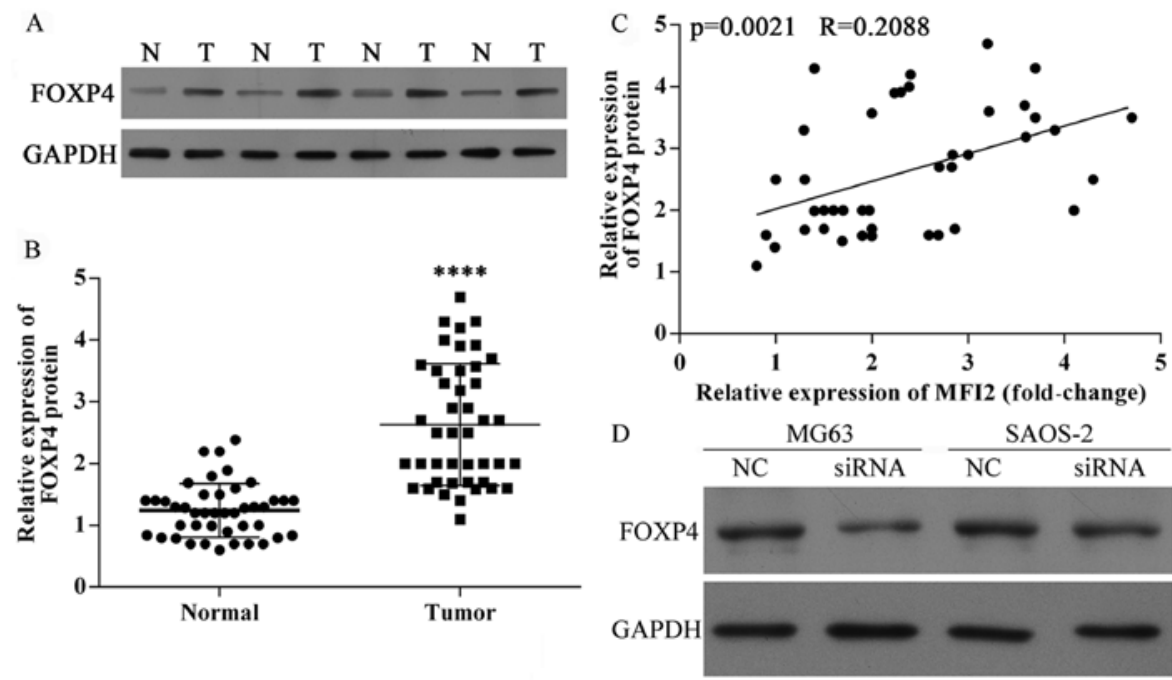

Figure 5. Correlation between MFI2 expression level and FOXP4 protein expression in osteosarcoma. (A) Western blot analysis of the protein expression of FOXP4 in both tumor samples (T) and normal tissues (N). (B) Quantification of the protein bands (OD ratio over GAPDH); ${ }^{* * *} \mathrm{P}<0.001$. (C) FOXP4 protein expression levels were positively correlated with MFI2 mRNA expression levels ( $\mathrm{P}=0.0021 ; \mathrm{R}=0.2088$ ). (D) The expression of FOXP4 in MG-63 and SAOS-2 cells after lncRNA MFI2-siRNA transfection.

A
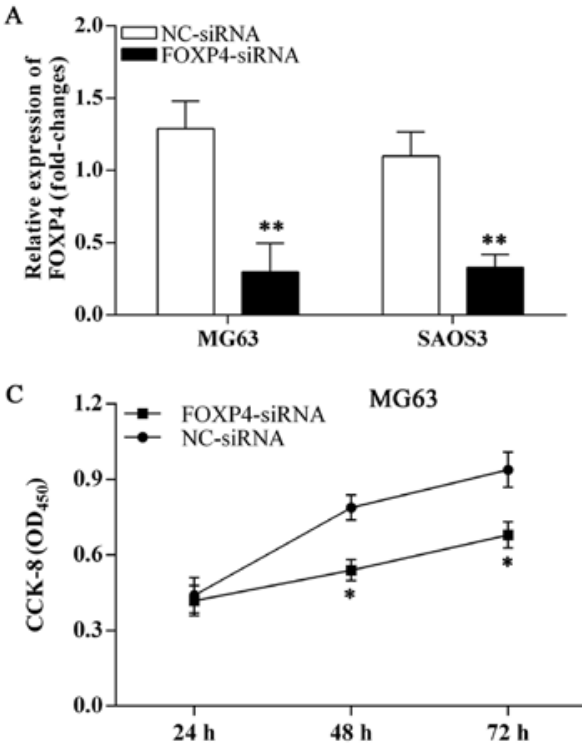

B
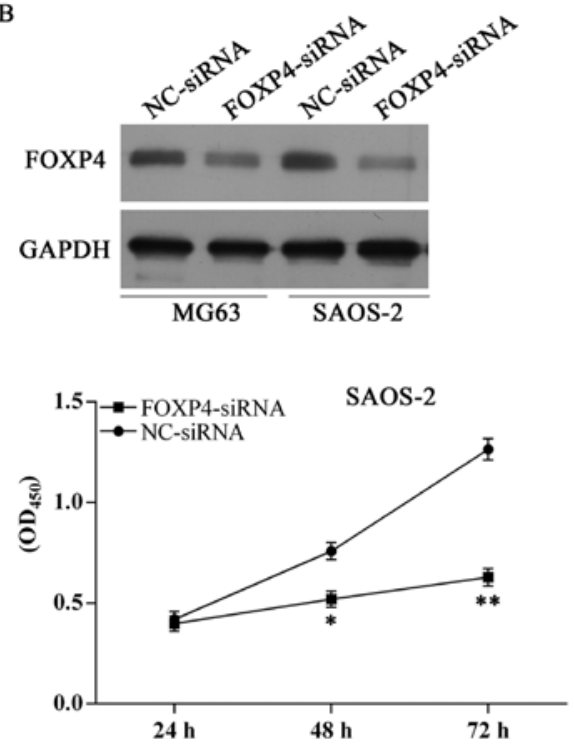

Figure 6. Knockdown of FOXP4 inhibits osteosarcoma cell proliferation. (A) The mRNA expression of FOXP4 in SAOS-2 and MG63 cells transfected with FOXP4-siRNA was detected using qRT-PCR, ${ }^{* *} \mathrm{P}<0.01$. (B) Western blot analysis the protein expression of FOXP4 in SAOS-2 and MG63 cells after FOXP4-siRNA transfection. (C) CCK-8 assay was used to detect proliferation rates of SAOS-2 and MG63 cells transfected with FOXP4-siRNA or NC; ${ }^{*} \mathrm{P}<0.05$, * $\mathrm{P}<0.01$.

Knockdown of MFI2 promotes osteosarcoma cell apoptosis in vitro. To confirm the effect of MFI2 on cell apoptosis, the Annexin V-FITC/PI staining method was performed to detec $\mathrm{T}$ cell apoptosis. The results showed apoptosis rates were increased in MG63 and SAOS-2 cells after MFI2-siRNA transfection (Fig. 4). The apoptosis rates of MG63 cells transfected with NC-siRNA and MFI2-siRNA were 11.6 \pm 2.4 and $45.1 \pm 6.4 \%$, respectively (Fig. 4). In addition, the apoptosis rates of SAOS-2 cells transfected with NC-siRNA and MFI2siRNA were $8.6 \pm 1.8$ and $30.8 \pm 5.4 \%$, respectively (Fig. 4).

FOXP4 protein is upregulated in osteosarcoma tissues and positively correlated with MFI2 expression. The expression of FOXP4 protein was analyzed by western blotting. It was observed that FOXP4 protein was significantly upregulated in osteosarcoma tissues, compared with matched normal tissues (Fig. 5A and B). As both the expression of MFI2 and FOXP4 were significantly enhanced, we considered a correlation between MFI2 expression and FOXP4 expression in osteosarcoma. FOXP4 protein expression levels were positively associated with MFI2 expression levels in 43 osteosarcoma tissues $(\mathrm{P}=0.0021 ; \mathrm{R}=0.2088$; Fig. 5C). Knockdown of lncRNA MFI2 expression suppressed the expression of FOXP4 (Fig. 5D).

Knockdown of FOXP4 suppresses osteosarcoma cell proliferation. To detect the role of FOXP4 on osteosarcoma cell proliferation, FOXP4 was knocked down by FOXP4-siRNA 
A

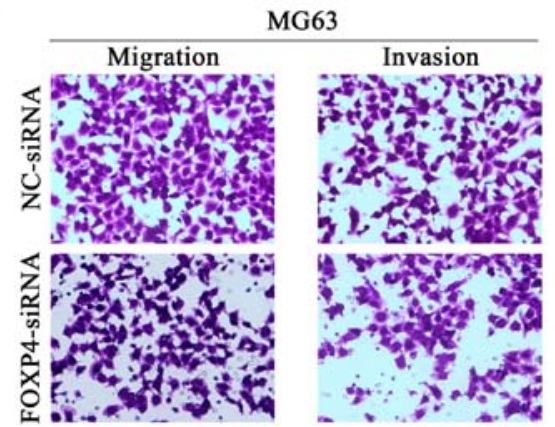

B

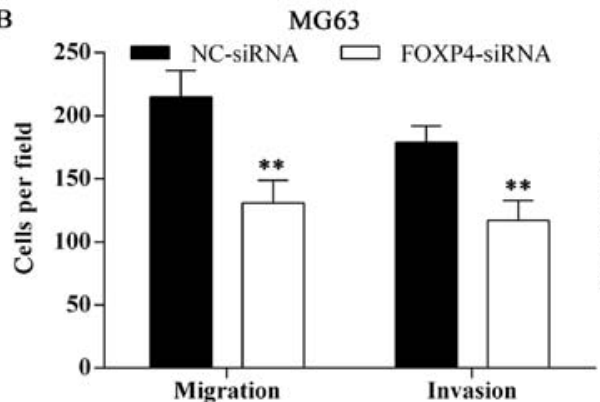

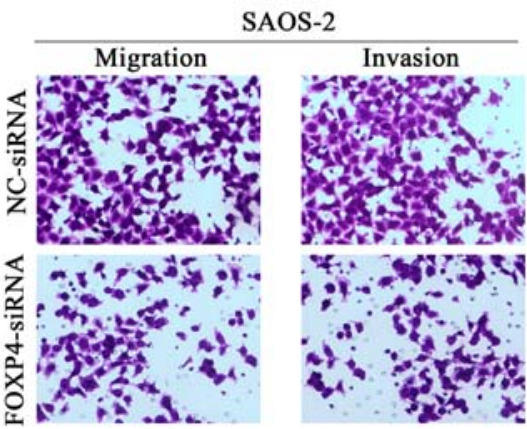

SAOS-2

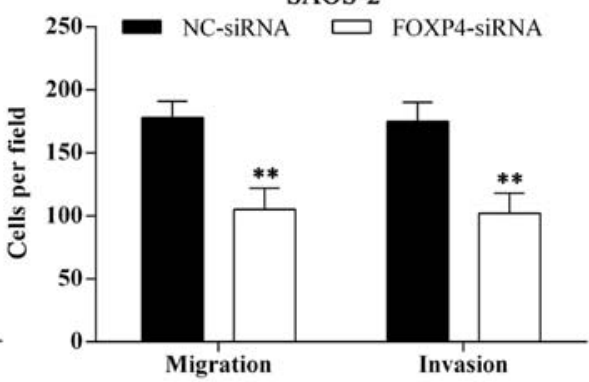

Figure 7. Knockdown of FOXP4 suppresses osteosarcoma cell migration and invasion. (A) Transwell assay was performed to detect SAOS-2 and MG63 cell migration and invasion (magnification, $\mathrm{x} 40$ ). Images of migration and invasion ability of each group are presented. (B) The average migration and invasion cell number per field among different experimental groups; ${ }^{* *} \mathrm{P}<0.01$.

A

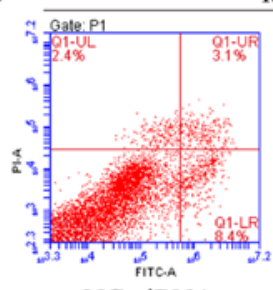

NC-siRNA
MG63

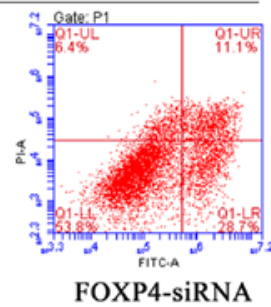

FOXP4-siRNA

B

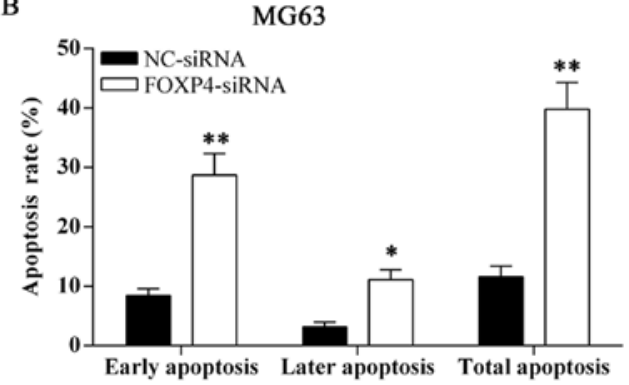

SAOS-2

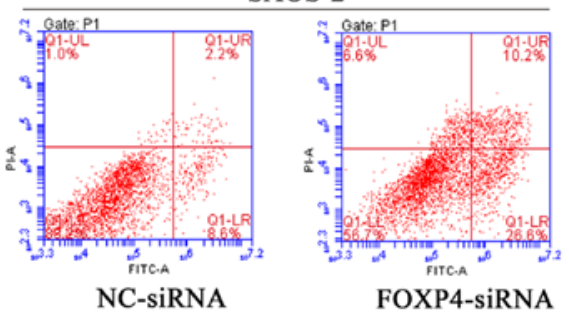

SAOS-2

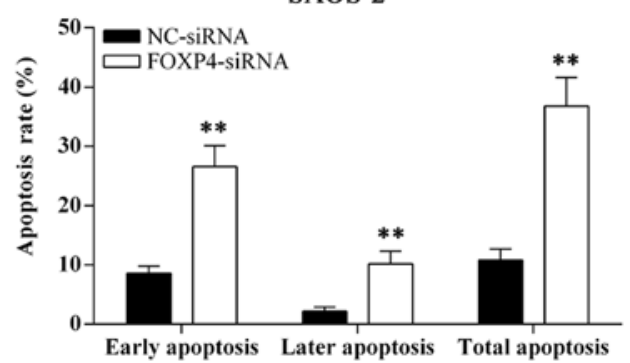

Figure 8. Knockdown of FOXP4 induces osteosarcoma cell apoptosis. Cell death was monitored by Annexin V-FITC/PI staining and flow cytometry. (A) Flow cytometric analysis of SAOS-2 and MG63 cells in different experimental groups. (B) The percentage of cells in early, later and total apoptosis of each group. Data are from three independent experiments; ${ }^{*} \mathrm{P}<0.05,{ }^{* *} \mathrm{P}<0.01$. LR (lower right), early apoptosis cells; UR (upper right), late apoptosis cells; LL (lower left), viable cells; UL (upper left), necrosis cells.

transfection. The expression of FOXP4 at mRNA and protein level was significantly decreased in MG63 and SAOS-2 cells after FOXP4-siRNA transfection (Fig. 6A and B). After FOXP4-siRNA transfection, osteosarcoma cell proliferation was significantly suppressed (Fig. 6C).

Knockdown of MFI2 inhibits cell migration and invasion. To confirm the effect of FOXP4 on cell migration and invasion, Transwell assay was used to detect the migration and invasion ability of MG63 and SAOS-2 cells after FOXP4-siRNA transfection. Knockdown of FOXP4 inhibited the migration and invasion ability of MG63 and SAOS-2 cells (Fig. 7).

Knockdown of FOXP4 promotes osteosarcoma cell apoptosis. To evaluate the impact of FOXP4 on cell apoptosis, the apoptosis assay was performed using Annexin V-FITC/PI staining method. The results showed apoptosis rates were increased in osteosarcoma cells after MFI2-siRNA transfection (Fig. 8). 
The apoptosis rates of MG63 cells transfected with NC-siRNA and MFI2-siRNA were $11.6 \pm 1.8$ and $39.8 \pm 4.5 \%$, respectively (Fig. 8). In addition, the apoptosis rates of SAOS-2 cells transfected with NC-siRNA and MFI2-siRNA were $10.8 \pm 1.9$ and $36.8 \pm 4.8 \%$, respectively (Fig. 8).

\section{Discussion}

In recent years, a series of studies found that numerous lncRNAs played important roles in fundamental cellular processes, such as promoting cellular growth and difference and affecting epigenetic information (19-21). It is increasingly recognized that the disorder of IncRNA expression involved in major pathologies, such as human cancer, resulting in progressive and uncontrolled tumor growth (22-24). Effective cell proliferation and survival intervention plays a critical role in preventing oncogenesis and treating cancer successfully. Therefore, increased understanding of lncRNA biological function may provide potential approaches for the diagnosis and treatment of human osteosarcoma.

In a previous study, the expression long non-coding RNA MFI2 was significantly upregulated in human osteosarcoma tissues (18). However, the exact role of MFI2 in evolution and progression of osteosarcoma is unknown. The present study was designed to investigate the role of MFI2 in occurrence and development of osteosarcoma. Our results showed that the expression of 1ncRNA MFI2 was significantly increased in osteosarcoma tissues and cell lines. In cellular functional studies, the results of CCK-8, Transwell and flow cytometric assays illustrated that knockdown of MFI2 by RNA interference markedly suppressed cell proliferation, migration and invasion in osteosarcoma cells, and promoted cell apoptosis. Furthermore, the expression of forkhead box P4 (FOXP4) was also detected using western blotting. The results showed FOXP4 was significantly overexpressed in osteosarcoma tissues, and FOXP4 protein expression levels were positively correlated with MFI2 expression levels. Taken together, our findings indicate that MIF2 could act as an oncogene through regulating cell growth, invasion, migration and apoptosis by regulating FOXP4 expression, and may be useful in diagnosis and treatment of osteosarcoma.

Forkhead box family transcription factors play important roles in cell type- and tissue-specific gene transcription regulation (25). FOXP4 as one of the FOXP subfamily members of winged-helix transcription factors, coding a 685-amino acid protein that is similar to FOXP1 and FOXP2 (26). During embryonic development, the expression of FOXP4 is observed primarily in gut, neural and pulmonary tissues (27). Wiehagen et al found that FOXP4 is expressed in immature thymocytes and mature T lymphocyte subsets and it is dispensable for T cell development. However, FOXP4 is required for normal $\mathrm{T}$ cell cytokine robust recall responses (28). In addition, Long et al successfully replicated the association of rs1983891 (FOXP4) with prostate cancer and provide further support for association of the FOXP4 with prostate cancer in Eastern Asian populations (29). Furthermore, the expression of FOXP4 was found increased in human hepatocellular carcinoma (HCC), knockdown of FOXP4 significantly suppressed HCC cell proliferation and colony formation in vitro by causing cell cycle arrest (30). However, the expression of
FOXP4 in osteosarcoma tissues and its role in evolution and progression of osteosarcoma are still poorly known. In the present study, our results indicated that FOXP4 expression was significantly increased in osteosarcoma tissues and cell lines. Knockdown of FOXP4 by FOXP4-siRNA transfection markedly suppressed cell proliferation, migration and invasion in osteosarcoma cells, and promoted cell apoptosis. All the results indicate that upregulation of lncRNA MFI2 may promote osteosarcoma cell proliferation through the FOXP4mediated signal pathway.

In conclusion, we demonstrated that lncRNA MFI2 and FOXP4 protein are upregulated in osteosarcoma tissues and cell lines, and FOXP4 protein expression levels were positively associated with MFI2 expression levels. Knockdown of MFI 2 and FOXP4 by RNA interference markedly suppressed cell proliferation, migration and invasion in osteosarcoma cells, and promoted cell apoptosis. Above all, lncRNA MFI2 was able to promote osteosarcoma cell proliferation by accelerating FOXP4, which indicated critical roles of MFI2 and FOXP4 in evolution and progression of osteosarcoma and may lead to new diagnostic and therapeutic approaches for osteosarcoma.

\section{References}

1. Picci P: Osteosarcoma (osteogenic sarcoma). Orphanet J Rare Dis 2: 6, 2007.

2. Caudill JS and Arndt CA: Diagnosis and management of bone malignancy in adolescence. Adolesc Med State Art Rev 18: 62-78, ix, 2007.

3. Walkley CR, Qudsi R, Sankaran VG, Perry JA, Gostissa M, Roth SI, Rodda SJ, Snay E, Dunning P, Fahey FH, et al: Conditional mouse osteosarcoma, dependent on p53 loss and potentiated by loss of $\mathrm{Rb}$, mimics the human disease. Genes Dev 22: 1662-1676, 2008.

4. O'Day K and Gorlick R: Novel therapeutic agents for osteosarcoma. Expert Rev Anticancer Ther 9: 511-523, 2009.

5. Bielack SS, Marina N, Ferrari S, Helman LJ, Smeland S, Whelan JS and Reaman GH: Osteosarcoma: The same old drugs or more? J Clin Oncol 26: 3102-3105, 2008.

6. Bertone P, Stolc V, Royce TE, Rozowsky JS, Urban AE, Zhu X, Rinn JL, Tongprasit W, Samanta M, Weissman S, et al: Global identification of human transcribed sequences with genome tiling arrays. Science 306: 2242-2246, 2004.

7. Guttman M, Amit I, Garber M, French C, Lin MF, Feldser D, Huarte M, Zuk O, Carey BW, Cassady JP, et al: Chromatin signature reveals over a thousand highly conserved large non-coding RNAs in mammals. Nature 458: 223-227, 2009.

8. Nagano $T$ and Fraser P: No-nonsense functions for long noncoding RNAs. Cell 145: 178-181, 2011.

9. Mercer TR, Dinger ME and Mattick JS: Long non-coding RNAs: Insights into functions. Nat Rev Genet 10: 155-159, 2009.

10. Cheetham SW, Gruhl F, Mattick JS and Dinger ME: Long noncoding RNAs and the genetics of cancer. Br J Cancer 108: 2419-2425, 2013.

11. Yang G, Lu X and Yuan L: LncRNA: A link between RNA and cancer. Biochim Biophys Acta 1839: 1097-1109, 2014.

12. Nie Y, Liu X, Qu S, Song E, Zou H and Gong C: Long noncoding RNA HOTAIR is an independent prognostic marker for nasopharyngeal carcinoma progression and survival. Cancer Sci 104: 458-464, 2013.

13. Niinuma T, Suzuki H, Nojima M, Nosho K, Yamamoto $H$, Takamaru H, Yamamoto E, Maruyama R, Nobuoka T, Miyazaki Y, et al: Upregulation of miR-196a and HOTAIR drive malignant character in gastrointestinal stromal tumors. Cancer Res 72: 1126-1136, 2012.

14. Li J, Yang S, Su N, Wang Y, Yu J, Qiu H and He X: Erratum to: Overexpression of long non-coding RNA HOTAIR leads to chemoresistance by activating the Wnt $/ \beta$-catenin pathway in human ovarian cancer. Tumour Biol 36: 9093-9094, 2015. 
15. Zhou X, Liu S, Cai G, Kong L, Zhang T, Ren Y, Wu Y, Mei M, Zhang $L$ and Wang $X$ : Long non-coding RNA MALAT1 promotes tumor growth and metastasis by inducing epithelial-mesenchymal transition in oral squamous cell carcinoma. Sci Rep 5: 15972, 2015.

16. Tripathi V, Shen Z, Chakraborty A, Giri S, Freier SM, Wu X, Zhang Y, Gorospe M, Prasanth SG, Lal A, et al: Long noncoding RNA MALAT1 controls cell cycle progression by regulating the expression of oncogenic transcription factor B-MYB. PLoS Genet 9: e1003368, 2013.

17. Ruan W, Wang P, Feng S, Xue Y and Li Y: Long non-coding RNA small nucleolar RNA host gene 12 (SNHG12) promotes cell proliferation and migration by upregulating angiomotin gene expression in human osteosarcoma cells. Tumour Biol 37: 4065-4073, 2016.

18. Li JP, Liu LH, Li J, Chen Y, Jiang XW, Ouyang YR, Liu YQ, Zhong H, Li H and Xiao T: Microarray expression profile of long noncoding RNAs in human osteosarcoma. Biochem Biophys Res Commun 433: 200-206, 2013.

19. Kotake Y, Nakagawa T, Kitagawa K, Suzuki S, Liu N, Kitagawa M and Xiong Y: Long non-coding RNA ANRIL is required for the PRC2 recruitment to and silencing of $p 15^{I N K 4 B}$ tumor suppressor gene. Oncogene 30: 1956-1962, 2011.

20. Liu Y, Zhao J, Zhang W, Gan J, Hu C, Huang G and Zhang Y: lncRNA GAS5 enhances G1 cell cycle arrest via binding to YBX1 to regulate p21 expression in stomach cancer. Sci Rep 5: 10159, 2016.

21. Su L, Han D, Wu J and Huo X: Skp2 regulates non-small cell lung cancer cell growth by Meg3 and miR-3163. Tumour Biol 37: 3925-393, 2015.

22. Hrdlickova B, de Almeida RC, Borek Z and Withoff S: Genetic variation in the non-coding genome: Involvement of microRNAs and long non-coding RNAs in disease. Biochim Biophys Acta 1842: 1910-1922, 2014.
23. Kung JT, Colognori D and Lee JT: Long noncoding RNAs: Past, present, and future. Genetics 193: 651-669, 2013.

24. Morris KV and Mattick JS: The rise of regulatory RNA. Nat Rev Genet 15: 423-437, 2014.

25. Chokas AL, Trivedi CM, Lu MM, Tucker PW, Li S, Epstein JA and Morrisey EE: Foxp1/2/4-NuRD interactions regulate gene expression and epithelial injury response in the lung via regulation of interleukin-6. J Biol Chem 285: 13304-13313, 2010.

26. Teufel A, Wong EA, Mukhopadhyay M, Malik N and Westphal H: FoxP4, a novel forkhead transcription factor. Biochim Biophys Acta 1627: 147-152, 2003.

27. Lu MM, Li S, Yang H and Morrisey EE: Foxp4: A novel member of the Foxp subfamily of winged-helix genes co-expressed with Foxp1 and Foxp2 in pulmonary and gut tissues. Gene Expr Patterns 2: 223-228, 2002.

28. Wiehagen KR, Corbo-Rodgers E, Li S, Staub ES, Hunter CA, Morrisey EE and Maltzman JS: Foxp4 is dispensable for T cell development, but required for robust recall responses. PLoS One 7: e42273, 2012.

29. Long QZ, Du YF, Ding XY, Li X, Song WB, Yang Y, Zhang P, Zhou JP and Liu XG: Replication and fine mapping for association of the C2orf43, FOXP4, GPRC6A and RFX6 genes with prostate cancer in the Chinese population. PLoS One 7: e37866, 2012.

30. Wang G, Sun Y, He Y, Ji C, Hu B and Sun Y: MicroRNA-338-3p inhibits cell proliferation in hepatocellular carcinoma by target forkhead box P4 (FOXP4). Int J Clin Exp Pathol 8: 337-344, 2015. 